\title{
Fractal Aspect of Iso-velocity Set in a Turbulent Boundary Layer*
}

\author{
Yoshiyuki TSUJI**, Katsuya HONDA***, \\ Hiroyuki TSUNODA** and Ikuo NAKAMURA**
}

\begin{abstract}
In this work, we report on the fractal aspect of an iso-velocity set in a turbulent boundary layer. The iso-velocity set is a one-dimensional point set defined by an instantaneous velocity signal. We use both a box-counting algorithm and a method based on the probability distribution function. The analysis is carried out carefully with clarification of the region of scale similarity. The iso-velocity set clearly indicates fractal features, except for near the local mean velocity, and their dimension is less than 0.4 in space. Scale similarity extends from the Kolmogorov scale to the largest eddy scale.The instantaneous turbulent energy field also shows fractal features and has a close connection with the iso-velocity set.
\end{abstract}

Key Words : Turbulent Flow, Boundary Layer, Iso-velocity Set, Fractal, Zerocrossing, Turbulent Energy

\section{Introduction}

Recently the concepts of fractals and chaos have been recognized as indispensable in the understanding of physical phenomena. Many studies have been reported in the recent past. In the field of mechanical engineering, for example, nonlinear oscillations and mixing processes are analyzed from the chaotic point of view. We hope the above concepts will be widely applied to many fields in science for a long time to come ${ }^{(1),(2)}$.

In this paper, we report the fractal aspect of an iso-velocity set in a turbulent boundary layer. The iso -velocity set, the definition of which will be given later, is a discrete time series and is obtained from the time series of instantaneous streamwise velocity signals. The same analysis has already been reported by Sreenivasan and Meneveau, but they did not point out clearly the region of scale similarity ${ }^{(3)}$. This is an important problem when we investigate fractals in

* Received 8th May, 1992. Paper No.90-1165A

** Department of Mechanical Engineering, Faculty of Engineering, Nagoya University, Nagoya 464-01, Japan

*** Department of Applied Physics, Faculty of Engineering, Nagoya University, Nagoya 464-01, Japan experimental data. We are concerned that their technique, the box-counting algorithm, tends to give a different result without distinctly defining the region of scale similarity ${ }^{(4)}$. Therefore we first investigate the fractal aspect of an iso-velocity set within a clearly defined region of scale similarity. Secondly we have tried to make out the spatial structure of the set, and studied the relationship between the instantaneous turbulence energy field.

The most well-known techniques for investigating the fractal structure are the box-counting algorith$\mathrm{m}$ (which is abbreviated as the B.C. method, hereafter), correlation function method, probability distribution function method (abbreviated as the P.D. method, hereafter), power spectrum method and so $\mathrm{on}^{(5),(6)}$. We made use of both the B. C. method and the P.D. method because of their easy calculation and accuracy.

As a result of analysis, we have come to the conclusion that the iso-velocity set clearly indicates fractal features, except for near the local mean velocity. Fractal dimensions are less than 0.4 and scale similarity extends from the Kolmogorov scale to the largest eddy scale. Another important result is that the instantaneous turbulent energy field also possesses fractal features. They have a close correlation with 
the iso-velocity set.

The fractal feature of turbulence is considered to be one of the geometrical aspects of chaos in turbulence. The above results indicate that there is a possibility of partially treating the property of turbulence as deterministic and contributory to the comprehension and prediction of turbulence.

\section{Nomenclature}

$\tilde{u}:$ instantaneous streamwise velocity

$U_{0}:$ mean streamwise velocity in free stream

$U(y)$ : local mean velocity at a point of $y$

$\delta:$ boundary layer thickness

$u:$ streamwise velocity fluctuation

$u^{\prime}:$ root mean square value of streamwise velocity

TH : threshold $\left(=\tilde{u} / U_{0}\right)$ defined by Eq. ( 1 )

$u_{t h}:$ threshold-velocity defined by Eq. (1)

$B_{t h}(t)$ : iso-velocity set defined by Eq. ( 2 )

$A(t)$ : turbulence energy set defined by Eq. (11)

$\tau_{k}:$ Kolmogorov time scale

$\tau_{\ell}:$ largest eddy scale

$P_{r}(\ell)$ : probability distribution function defined by Eq. ( 6 )

$D_{B}$ : fractal dimension calculated by B. C. method

$D_{P}$ : fractal dimension calculated by P. D. method

$D_{d}$ : fractal dimension of turbulent energy field

$D$ : fractal dimension (a general term of noninteger dimension)

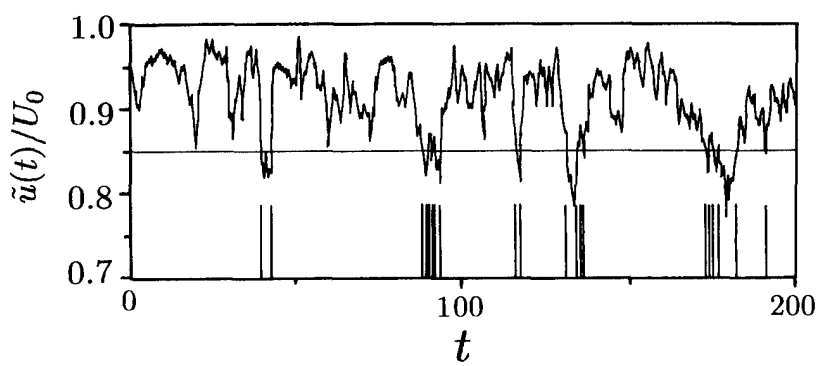

Fig. 1(a) Instantaneous streamwise velocity signal and iso-velocity set at the point $y / \delta=0.56$. Solid line express the threshold value

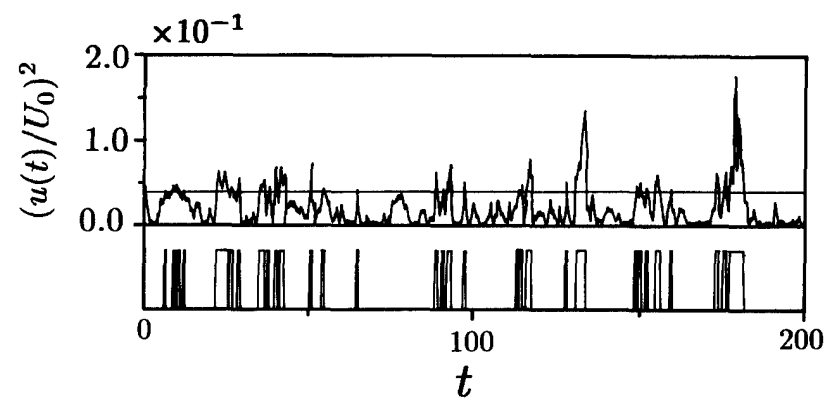

Fig. 1( b ) Distribution of streamwise instantaneous turbulent energy field at the point $y / \delta=0.56$. Solid line is a time-average value of instantaneous turbulent energy

\section{Analytical Conditions and Methods}

\subsection{Iso-velocity set}

An outline of the streamwise velocity signal and the iso-velocity set are shown in Fig. 1 (a). Figure 1 (b) indicates the distribution of instantaneous turbulent energy fields, which will be explained in section 3. 4. In Fig. 1 (a) the vertical axis is a dimensionless quantity and the horizontal axis has the dimension of time. The horizontal solid line is called a threshold and we express it as $T H$ hereafter. For instance, $T H$ $=\tilde{u} / U_{0}=0.85$ in the figure. The iso-velocity set is defined as the intersection of $\tilde{u} / U_{0}$ and $T H$. The positions of vertical lines indicate the elements of the iso-velocity set. In particular, the instantaneous streamwise velocity determined by $T H$ is called a threshold-velocity, denoted as

$$
u_{t h}=U_{0} \cdot T H \text {. }
$$

For later analysis, we define the iso-velocity set as

$$
B_{t h}(t)=\left\{t \mid u_{t h}=\tilde{u}(t)\right\} \text {. }
$$

In this analysis, the data length which is used for computation is called a segment length. It is also one of the important parameters for fractal analysis.

Experimental data were obtained from a twodimensional turbulent boundary layer with no pressure gradient. The I-type hot wire probe was set at $1900 \mathrm{~mm}$ downstream from the leading edge of the plate and was operated by a constant-temperature anemometer. The signal from the anemometer was recorded in digital format with the frequency $5 \mathrm{kHz}$. Characteristics of our boundary layer are as follows : external flow velocity $U_{0}=5.0(\mathrm{~m} / \mathrm{s})$, boundary layer thickness $\delta=40.0(\mathrm{~mm})$ and Reynolds number $\operatorname{Re}_{\delta}=$ $U_{0} \delta / \nu=1.4 \times 10^{4}$. The Kolmogorov time scale ${ }^{(7)}$ and the largest eddy scale are estimated to be $\tau_{k} \sim 1.5(\mathrm{~ms})$ and $\tau_{\ell} \sim 20.0(\mathrm{~ms})$, respectively. We evaluate the largest eddy scale to be equal to the value at which the autocorrelation coefficient of the streamwise velocity crosses the time axis for the first time.

\subsection{Box counting algorithm}

We will briefly explain the B.C. method. The segment length is equally divided into small boxes with equal length, $\varepsilon . N(\varepsilon)$ is the number of boxes which contain at least one element of $B_{t h}$. Therefore the iso-velocity set is covered (or approximated) by $N(\varepsilon)$ boxes. When we change the box size, the following equation,

$$
N(\varepsilon) \propto \varepsilon^{-D_{s}},
$$

is always valid for a fractal set. The exponent $D_{B}$ is called the box dimension ${ }^{(5)}$. Although its original definition can be exactly given by a measure theory ${ }^{(8),(9)}$, in this analysis we assume that $D_{B}$ is an exponent for the evaluation of the statistical selfsimilarity of a figure. It is possible to consider it as an 
extension of the similarity dimension ${ }^{(5)}$. The original box dimension can be defined, for example, for the Cantor set or the Koch curve. On the other hand, the exponent $D_{B}$ in Eq. ( 3 ) is computed for a coastline or a cloud which has statistical self-similarity.

Figure 2 shows a typical example of analyzing the iso-velocity set by means of the B.C. method. The solid line has a slope of -1 . This means that the isovelocity set is regarded as a line with this box size. The slope of the broken line gives the fractal dimension $D_{B}$. The span between the two arrows on the horizontal axis corresponds to the region of scale similarity, that is, [ $\left.\varepsilon_{\min }, \varepsilon_{\max }\right]$. The slope is calculated within this region by the least mean squares approximation. Fractals that are recognized in nature should have this finite scale similarity region. Here the exponent $D_{B}$ defined in Eq. ( 3 ) is a function of [ $\varepsilon_{\min }$, $\left.\varepsilon_{\max }\right]^{(4)}$, but the previous analysis was carried out without clearly indicating this region ${ }^{(3)}$. When we discuss the value of $D_{B}$ quantitatively, the scale similarity region should be presented. In our analysis this is defined as follows and the iso-velocity set is investigated within this region.

The smallest box size $\varepsilon_{\min }$ corresponds to the Kolmogorov time scale $\tau_{k}$, because the smaller size does not have a physical meaning for the turbulent eddy. Here $\varepsilon_{\max }$ is determined empirically :

$$
\varepsilon_{\max }=A V E+R M S / 2 \text {, }
$$

where $A V E$ and $R M S$ are the average and r.m.s. values of time intervals between each element of the iso-velocity set, respectively. If the distribution of intervals is supposed to be the Poisson process, the probability that the interval length is less than $\varepsilon_{\max }$ is 0.777 and if the distribution is Gaussian, its probability is 0.691 .

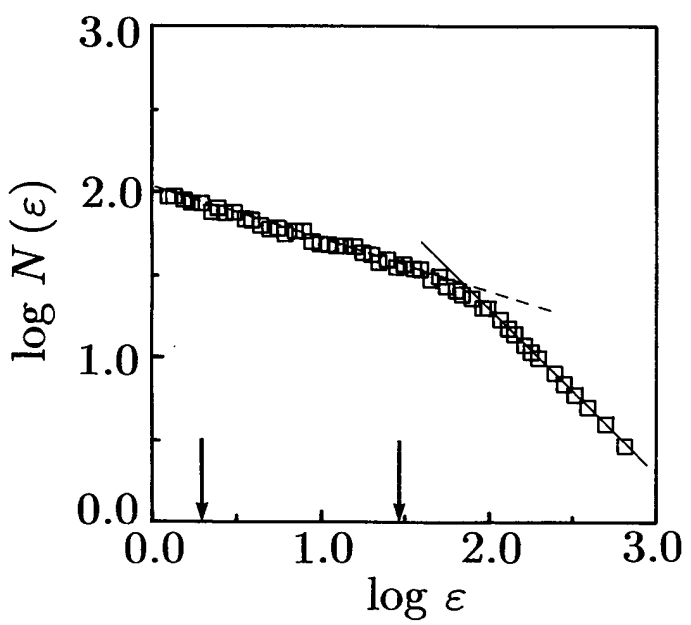

Fig. 2 One of the example of computing iso-velocity set by B. C. method. Iso-velocity set is defined at $y / \delta=$ 0.70 and $T H=0.86$
We introduce the correlation coefficient $R$ that evaluates the accuracy of the least mean squares approximation $^{(10)}$. When $|R|=1$, its approximation is a straight line. In this analysis we exclude data whose correlation coefficients are less than 0.97 .

\section{3 Method of probability distribution function}

The other method for fractal analysis is the method of the probability distribution function ${ }^{(5)}$. First of all, we define $L$ and $\ell$ as a random variable and a state variable of intervals, respectively. Generally, the probability distribution function, $P(\ell)$, refers to the probability prob $\{L \leq \ell\}$, but we use the following definition here for convenience.

$$
P_{r}(\ell)=1-P(\ell)
$$

If the iso-velocity set has a fractal feature, $P_{r}(\ell)$ satisfies the following relationship for various $\ell$ :

$$
P_{r}(\ell) \propto \ell^{-D_{p}}
$$

where $D_{P}$ is the fractal dimension ${ }^{(5)}$.

The scale similarity relationship is only possible for a power law distribution of $\operatorname{Pr}_{r}(\ell)$. We consider that the statisfical self-similarity is realized when Eq. ( 6 ) is satisfied for some $\ell$ values. In Fig. 3 we show a typical example of analyzing the iso-velocity set by this probability distribution function. The symbol, $\square$, shows experimental results. It is clear that a straight part remains between the two arrows on the axis. These arrows indicate the region, $\left[\ell_{\min }, \ell_{\max }\right]$, where scale similarity is realized. In our investigation, $\ell_{\min }=$ $\tau_{k}$ and $\ell_{\max }=\tau_{\ell}$, respectively. From Eq. (6), the slope of the broken line is equal to the exponent $D_{P}$. It is calculated by the least mean squares approximation and as in the B.C. method we omit the data whose correlation coefficients are less than 0.97 .

We also compute the Poisson process and indicate its result with the symbol $\times$ in Fig. 3 . It is curved and no straight part remains on it.

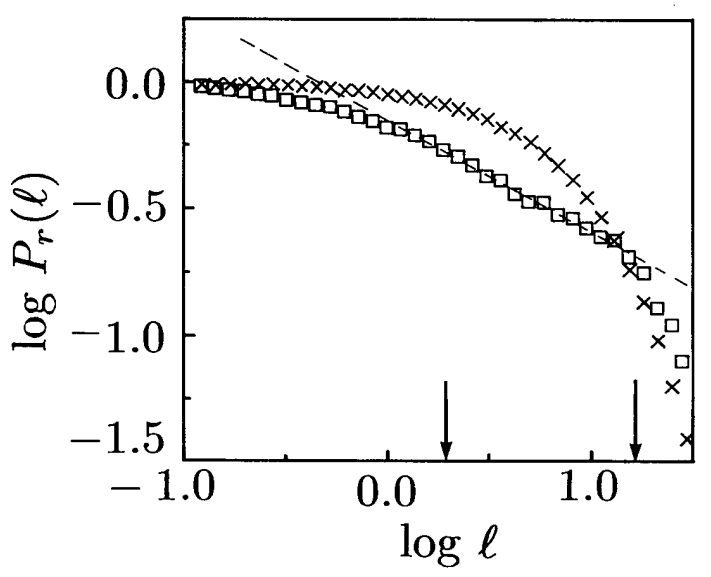

Fig. 3 One of the examples of computing iso-velocity set by P. D. method. Iso-velocity set is defined at $y / \delta=$ 0.70 and $T H=0.88$. The symbol $\square$ is an experimental result and $x$ is the Poisson distribution 


\section{4 Effect of segment length}

Segment length is an important parameter in the analyses of the B.C. method and the P. D. method. Figure 4 and Table 1 show the effect of segment length upon fractal dimensions. The data in Fig. 4 seem to show an overall curvature when the segment length gets longer. Howeverr, fractal dimensions calculated by the B. C. method within the range $\left[\varepsilon_{\min }\right.$, $\left.\varepsilon_{\max }\right]$ are not influenced by the segment length. Then in later analysis, we fix the segment length at $2.0 \times 10^{3}$ (ms) ; about one hundred times larger than the largest eddy scale, in order to obtain a sufficient number of iso-velocity points, within the segment. The maximum value $\varepsilon$ is slightly scattered but it has a unique value for a sufficiently long length segment.

Although the same analysis was done in Ref. ( 3 ) (p. 373, Fig. 14), it appears to be vague. It is reported that a short segment length (about fifty times as large as the transverse integral scale) gives a distinct constant-slope region on the graph. On the contrary, a

Table 1 The relationship between fractal dimension and segment length

\begin{tabular}{|c|rr|c|c|}
\hline & \multicolumn{2}{|c|}{ Segment } & $D$ & $R$ \\
\hline$\square$ & 200 & $(m s)$ & 0.44 & -0.97 \\
\hline$\bigcirc$ & 600 & $(m s)$ & 0.45 & -0.99 \\
\hline$\triangle$ & 1000 & $(m s)$ & 0.43 & -0.99 \\
\hline+ & 1400 & $(m s)$ & 0.43 & -0.99 \\
\hline$\diamond$ & 2200 & $(m s)$ & 0.43 & -0.99 \\
\hline
\end{tabular}

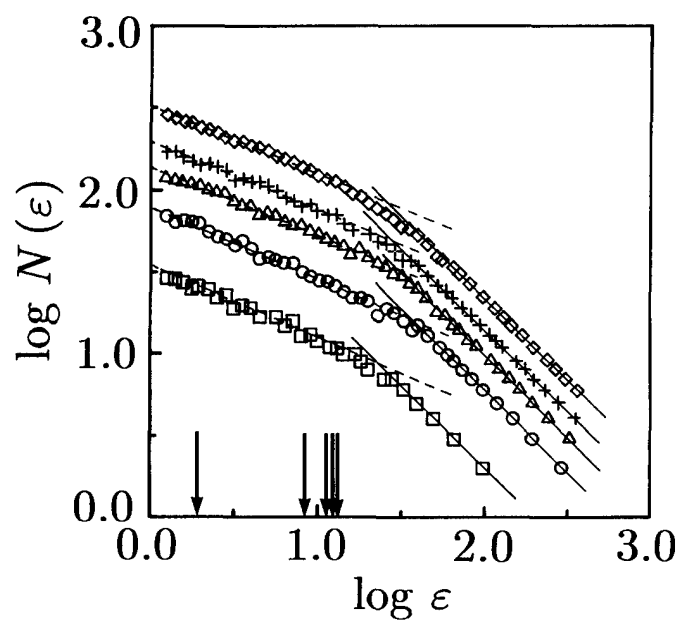

Fig. 4 The relationship between fractal dimension and segment length. Different symbols express the results computed by the B.C. method with different segment lengths. The detailed definition of symbols is listed in Table 1 large segment length (about four hundred and thirty times larger) leads to the result of a less well-defined constant-slope region on the graph. Then they concluded that the iso-velocity set indicates the fractal feature only in the case of a short segment length. However, we cannot easily distinguish the constantslope region on the graph from the less well-defined constant-slope region on the graph. That is because the scale similarity region is not clearly indicated.

\section{Results and Discussion}

\section{1 Fractal dimensions computed by the two methods}

For the one-dimensional fractal point set, $D_{B}$ (defined in Eq. (3)) and $D_{P}$ (defined in Eq. (6)) approximately ${ }^{(11)}$ coincide. Hereafter we express them as $D$. In Fig. 5, we plot the fractal dimensions computed by the two methods when the threshold is changed in the case of three representative wall-normal positions. The symbols $\square$ and $\times$ show results of the $B$. $C$. method and P.D. method, respectively. The parameter $P$ is defined as follows:

$$
P=\frac{T H-U(y) / U_{0}}{u^{\prime} / U_{0}}=\frac{u_{t h}-U(y)}{u^{\prime}},
$$

where $U(y)$ and $u^{\prime}$ are local mean velocity and r. m. s. value at the position $y$, respectively. When $P$ equals zero, the threshold-velocity is equal to local mean velocity $U(y)$. We call this particular threshold a zerocrossing ${ }^{(12)}$. Fractal dimensions computed by each technique are widely different around the zero-crossing. On the other hand, they coincide well when $|P| \geq$ 1.5 and their values are $D \leq 0.40$. It is also noted that $\varepsilon_{\max }$ becomes as large as the largest eddy scale in the region where $D_{B}=D_{P}$; that is, $|P| \geq 1.5$. This characteristic holds both in the wall region and in the outer region of the turbulent boundary layer. We will consider the reason why fractal dimensions are different around the zero-crossing from the phenomenological point of view in the next section.

In Fig. 6, we show the fractal dimensions with a fixed threshold-velocity across the boundary layer. No dimension of each threshold-velocity is constant in space. The dashed line express the position where the fixed threshold-velocity equals the local mean velocity. This is also equivalent to the place of the zero-crossing. Fractal dimensions have their maximum values in the vicinity of local mean velocity but gradually decrease away from it. It is remarkable that the fractal dimensions computed by the B. C. method and the P. D. method agree well, except near the local mean velocity.

\section{2 Iso-velocity set at the zero-crossing}

Judging from the result that the fractal dimensions computed by the two methods do not agree well 


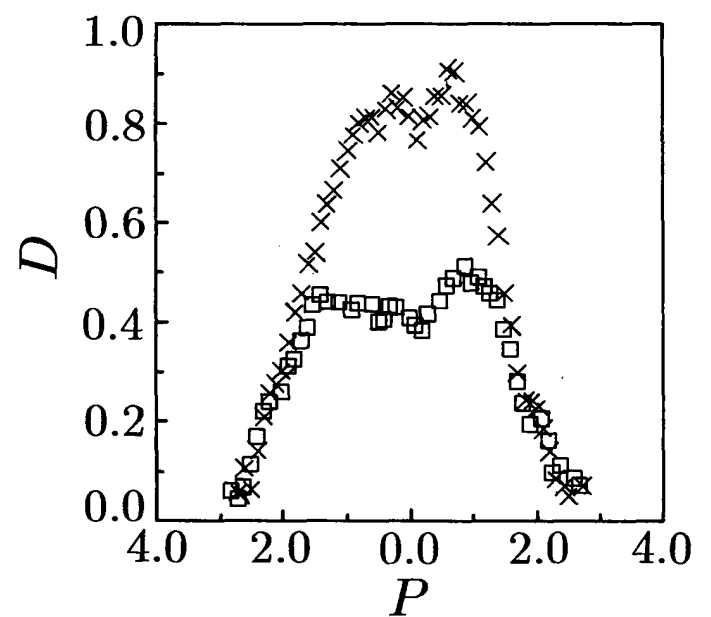

(a) Fractal dimensions computed by two methods near the wall region, $y^{+}=21.8$. The symbol $\square$ is the $B$. C. method and $x$ is the P. D. method

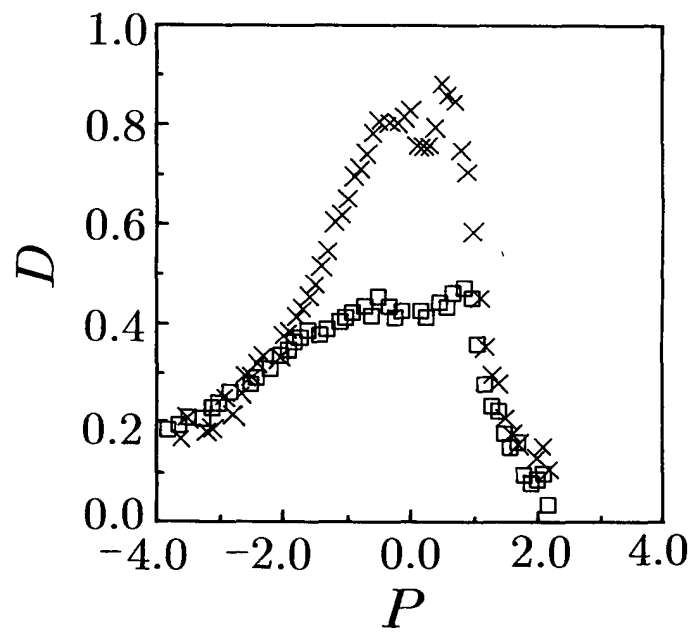

(b) Fractal dimensions computed by two methods at the log-law regions, $y^{+}=104.5$. The symbol $\square$ is the B. C. method and $\times$ is the P. D. method

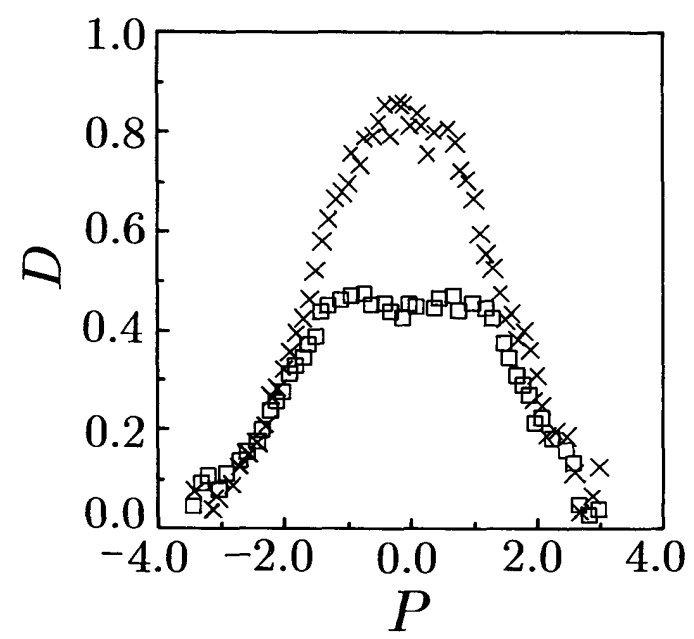

(c) Fractal dimensions computed by two methods at outer region, $y^{+}=436.7$. The symbol $\square$ is the B. C. method and $x$ is the P.D. method

Fig. 5 The change of fractal dimensions for the parameter $P$ in the case of three representative points in turbulent boundary layer around the zero-crossing, we assume that the isovelocity set loses its fractal character around that position. The previous study has reported similar results, but detailed analysis was not done $e^{(3)}$. We will attempt to consider phenomenologically the iso-velocity set at the zero-crossing. In Fig. 7, we show one of the examples of computing the iso-velocity set at the zero-crossing by two methods. Here $\varepsilon_{\max }$, which is defined by Eq. ( 4 ), empirically has a small value near the zero-crossing, because the instantaneous velocity signal crosses the threshold most frequently there, so that $A V E$ decreases. The self-similarity range $\left[\varepsilon_{\mathrm{min}}\right.$, $\left.\varepsilon_{\max }\right]$, becomes small and it is difficult to confirm the straight line part between the two arrows. In the results of the P.D. method, the experimental data agree well with those of the Poisson process.

In order to comprehend the zero-crossing isovelocity sets, we will introduce the concept of the iso -velocity surface. The threshold-velocity is fixed and
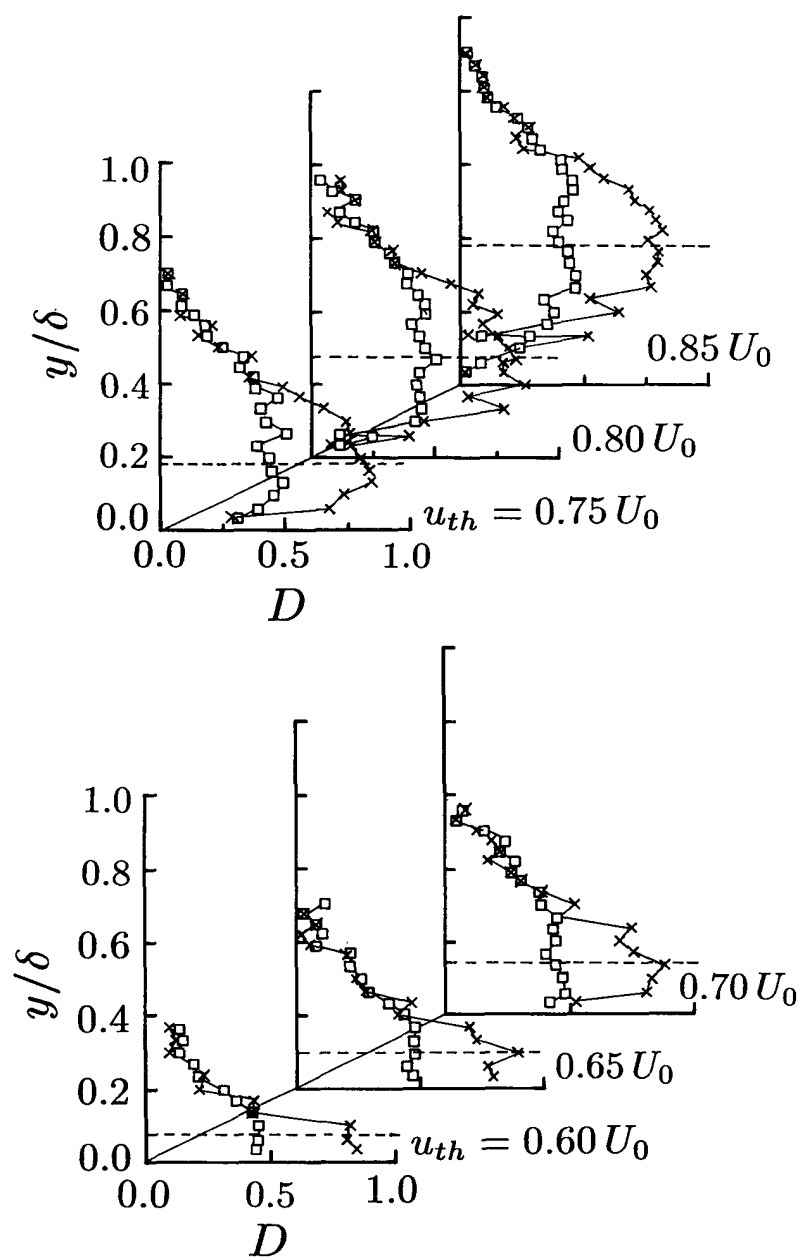

Fig. 6 The change of fractal dimensions with fixed threshold-velocity in space. The dashed line express the position where local mean velocity equals threshold-velocity. The symbol $\square$ is the B. C. method and $x$ is the P. D. method 
we consider the points whose instantaneous velocity is equal to $u_{t h}$.

$$
\tilde{u}(x, y, z, t)=u_{t h}
$$

It is supposed that these points are located on the surface which have a bubble-like structure with the thickness of the Kolmogorov scale. These surface are sketched schematically in Fig. 8, when we cut them in the $x-y$ plane. The curves are drawn by Brownian motion for the sake of convenience. In Fig. 8, the $Z_{0}$ axis express the location where the local mean velocity $U(y)$ equals $u_{t h}$, and also stands for the zerocrossing. Intersections of iso-velocity surfaces and a straight line which is parallel to the $Z_{0}$ axis are equivalent to the iso-velocity set defined in Eq. ( 2 ) if we use Taylor's hypothesis.

In the course of experimental analysis, it is clear that the number of elements $N$ of the iso-velocity set

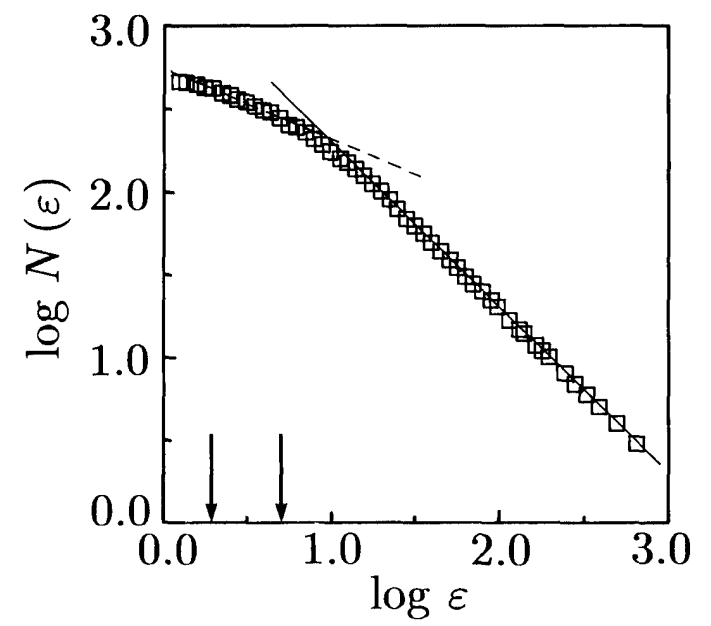

(a) The result of computing the iso-velocity set at zerocrossing by the B. C. method at $y / \delta=0.42$

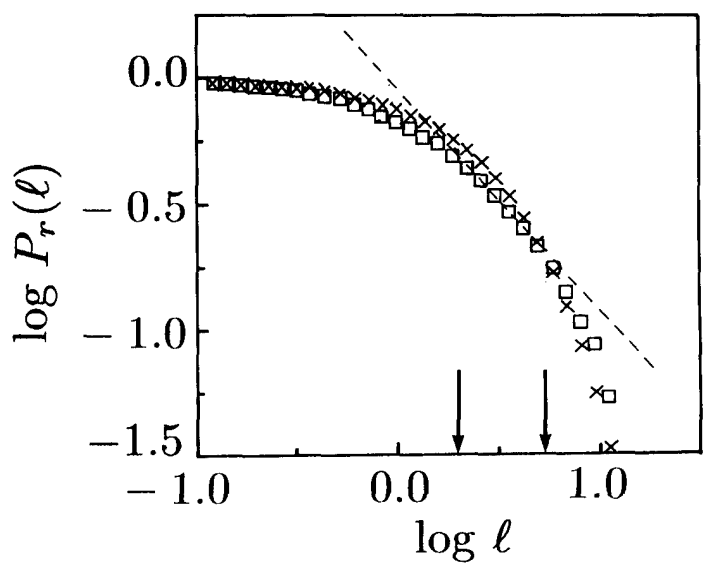

(b) The result of computing the iso-velocity set at zerocrossing by the P. D. method at $y / \delta=0.42$. The symbol $\square$ is an experimental result and $X$ is the Poisson distribution

Fig. 7 The analysis of the iso-velocity set at zerocrossing whose instantaneous velocity is equal to $u_{t h}$, indicates its maximum value, $N_{0}$, near the local mean velocity. But $N$ decreases gradually as points which are far from the $Z_{0}$ axis. This is also equivalent to the fact that the instantaneous velocity crosses most frequently near the zero-crossing. Considering that the fluctuation of the streamwise velocity is finite, and the local mean velocity is not constant in the turbulent boundary layer, iso-velocity surfaces become sparse at the distance from the $Z_{0}$ axis. As seen in Fig. 8, iso-velocity surface may be complicated and cross one another near the zero-crossing, but isolated surfaces can exist apart from it.

If the iso-velocity surface has a fractal structure, does the iso-velocity set also have a fractal structure? This question has not yet been answered. Let us consider this problem using a simple example. A onedimensional Brownian motion produces a random cantor set on the zero-crossing, whose fractal dimension is $0.5^{(5)}$. But as sketched in Fig. 8 , the random cantor set produced by the intersections of several Brownian motions and the zero-crossing seems to lose its fractal feature. Figure 9 is the result of computing the points produced by several Brownian motions on the zero-crossing. No straight region remains in the figure.

It is natural to think that the reason why the P. D. method and the B. C. method did not give the same fractal dimension for the zero-crossing iso-velocity set is that several iso-velocity surfaces exist near the zero-crossing.

Some studies report that the distribution of $\ell$ in Eq. ( 5 ) has an exponential form ${ }^{(12)}$ at the zero-crossing and some report a lognormal distribution ${ }^{(13)}$. The number of zero-crossing per second, $N_{0}$, is related to Taylor's microscale, fine-structure and vorticity distributions ${ }^{(12),(13)}$. Sreenivasan et al. studied the probability density function of $\ell$ and concluded that the distribution of a large eddy is different from that of a small eddy ${ }^{(12)}$. It is also interesting that the scale he established to distinguish the large scale eddy from the small one is equal to $\varepsilon_{\max }$ in Eq. (4).

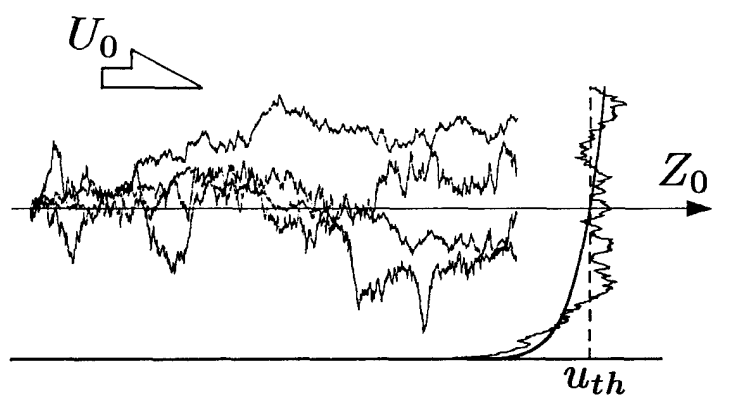

Fig. 8 Schematic view of iso-velocity surfaces around the zero-crossing 


\section{3 Change of fractal dimensions}

As shown in Figs. 5 and 6, the fractal property is inherent in the iso-velocity set, except near the zerocrossing, but their fractal dimensions are not constant. This seems to contradict the fact that the iso-velocity surface has a unique fractal dimension. We shall now look more carefully into this problem.

Figure 10 is a fractal curve which is constructed by a generator indicated in the inset ${ }^{(14)}$. We will regard this curve as an example of an iso-velocity surface, $S$. If this surface has a fractal dimension (Hausdorff dimension) $\operatorname{dim}_{H}(S)$, its intersection with a straight line, $m$, is also fractal. The fractal dimension $\operatorname{dim}_{H}(S \cap m)$ satisfies the following relationship ${ }^{(9)}$ :$$
\operatorname{dim}_{H}(S \cap m) \leq \max \left\{0, \operatorname{dim}_{H}(S)+\operatorname{dim}_{H}(m)-n\right\},
$$

where $n$ is a topological dimension of support. Thus we have

$$
\operatorname{dim}_{H}(m)=1, n=2 .
$$

Fractal dimension $\operatorname{dim}_{H}(S \cap m)$ changes according to the manner in which the straight line $m$ intersects the surface $S$, even if $S$ has a unique dimension. As mentioned previously, Taylor's hypothesis makes it possible to regard the set $\{S \cap m\}$ as $B_{t h}(t)$.

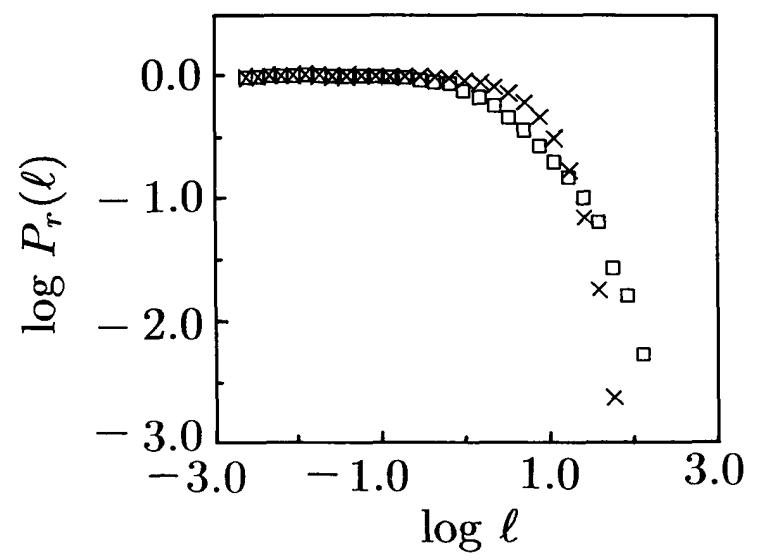

Fig. 9 The result of computing the iso-velocity set produced by several Brownian motions at zerocrossing by means of P.D. method. The symbol $\square$ is a result of the Brownian motion and $x$ is the Poisson process

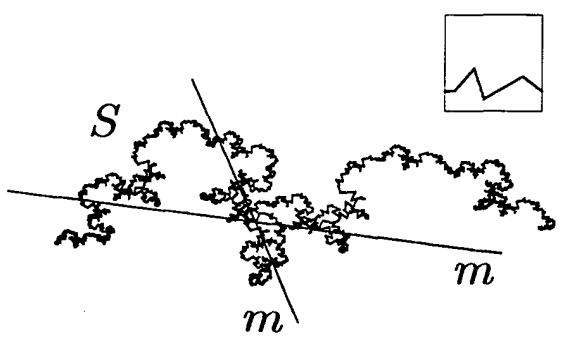

Fig. 10 Schematic view of iso-velocity surface and its generator
Close to the zero-crossing region, the iso-velocity set loses its fractal feature because several iso-velocity surfaces may cross one another. But far from the zero-crossing, there are isolated surfaces. We can compute their fractal dimensions by means of the B. C. method and the P.D. method. Their values gradually decrease away from the zero-crossing. This change of fractal dimensions is able to be comprehended by the concept of "intersections of fractal sets"(9).

It is generally supposed that the fractal character of turbulence may be closely related to the distribution of eddies. Large eddies break into small eddies and small ones break into still smaller ones. This process is considered to be self-similar. Thus it is natural to think that the scale similarity extends from the smallest eddy scale to the largest one. Actually in this analysis, when the B. C. method and P. D. method give the same fractal dimension, the scale similarity range is equal to $\left[\tau_{k}, \tau_{\ell}\right]$. This is independent of flow patterns.

\section{4 Relationship between iso-velocity set and turbulent energy field}

Here we will investigate the turbulent energy field from a fractal point of view. First of all, we define the instantaneous turbulent energy by $u(t)^{2}$, where $u(t)$ is the fluctuation of the instantaneous streamwise velocity. Usually, turbulent energy, $\overline{u(t)^{2}}$, is the time average of $u(t)^{2}$. Secondly, we define the one-dimensional set $A(t)$ as follows :

$$
A(t)=\left\{t \mid u(t)^{2} \geq \overline{u(t)^{2}}\right\} .
$$

Instantaneous turbulent energy is shown in Fig. 1( b ). The solid line indicates the value of $\overline{u(t)^{2}}$. The series of pulses are regions where instantaneous turbulent energy is larger than its time average value, that is, $A(t)$.

Using the parameter $P$ in Eq. ( 7 ), the threshold -velocity is also expressed as

$$
u_{t h}=U(y)+P \times u^{\prime} \text {. }
$$

We will now define $B(t)$, using the above $u_{t h}$, as

$$
B(t)=\left\{t\left|B_{t h}(t), 1.5 \leq\right| P \mid\right\} \text {. }
$$

The set $B(t)$ is equivalent to all of the iso-velocity sets in which both the B.C. method and the P.D. method give the same fractal dimension. From Eq. (11) to Eq. (13) we can easily find

$$
B(t) \subset A(t) \text {. }
$$

We analyze the fractal character of the above set $A(t)$ by the B.C. method. One of the examples is shown in Fig. 11. The straight region remains in the figure and this indicates that $A(t)$ has a fractal property. The slope of the dotted line gives a fractal dimension $D_{d}$. The scale similarity region is set within $\left[\tau_{k}\right.$, $\tau_{\ell}$ ]. So far it has not yet been clarified theoretically that the turbulent energy field has a fractal distribu- 


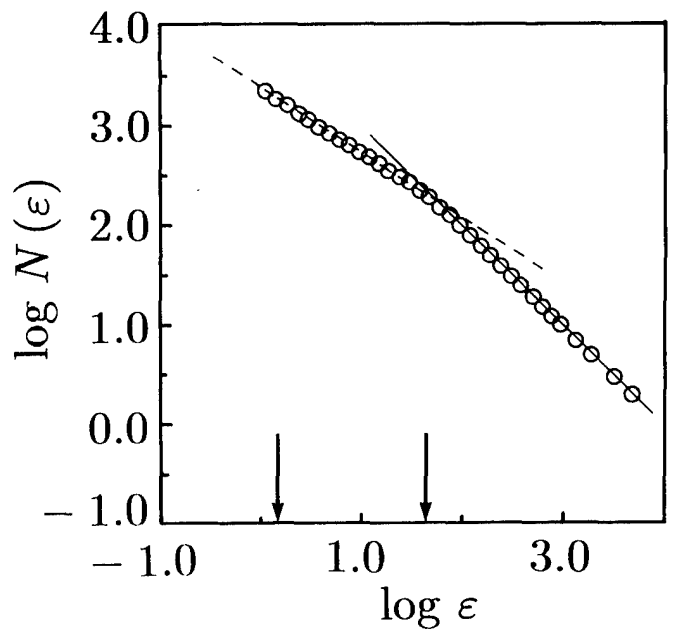

Fig. 11 One of the examples of computation of the set $A(t)$ by the B. C. method. $A(t)$ is defined in Eq. (11) at $y / \delta=0.78$

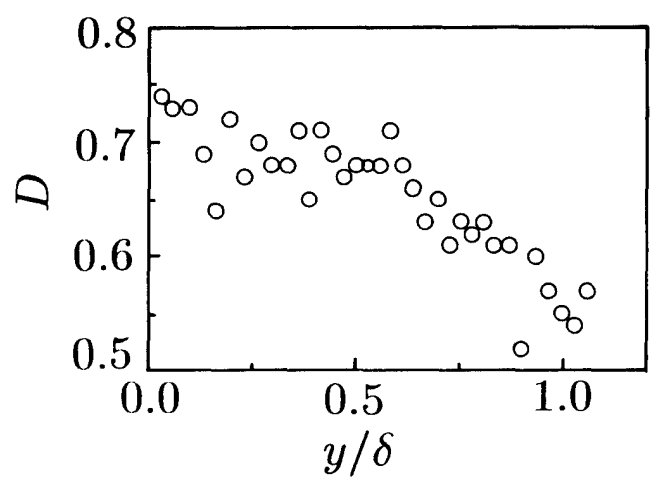

Fig. 12 Fractal dimensions of the set $A(t)$ in the turbulent boundary layer

tion. Moreover, no turbulence model which uses this result has been contructed, to the best of our knowledge.

Since the fluctuation of velocity is largest near the wall region, $A(t)$ should have a complicated structure. We can evaluate $A(t)$ qualitatively by fractal dimensions and the result is shown in Fig. 12. The fractal dimensions decrease in the outer region of the turbulent boundary layer.

\section{Conclusions}

We analyzed the iso-velocity sets in the turbulent boundary layer, with clarification of the scale similarity region. Both the B. C. method and the P. D. method were used for computation and their results were compared with each other. We have come to the following conclusions.

1. In the turbulent boundary layer, the iso-velocity set clearly has a fractal structure except in the region close to the local mean velocity. Scale similar- ity extends from the Kolmogorov time scale, $\tau_{k}$, to the largest eddy scale, $\tau_{\ell}$. Their fractal dimensions are not unique and less than 0.4 . In our analysis, the fractal property of the iso-velocity set is independent of segment length.

2. The regions where the instantaneous turbulent energy is larger than its average value, $A(t)$, contain all iso-velocity sets, which clearly indicate fractal feature, that is, $B(t) . A(t)$ also has self-similarity and we can evaluate its structure quantitatively by fractal dimensions. $A(t)$ may be the most complicated near the wall because of bursting phenomena. The fractal dimension of $A(t)$ is largest near the wall but decreases away from it in the turbulent boundary layer.

The experimental data were measured with the help of Mr. K. Okumura. We wish to express our gratitude to him.

\section{References}

(1) Iida, S., On the Publication of a Special Issue on "Chaos", Trans. Jpn. Soc. Mech. Eng., (in Japanese), Vol. 91, No. 840, (1988), p. 1.

(2) Francis, M., Chaos, Mech. Eng., Vol. 112, No.1, (1990), p. 40.

(3) Sreenivasan, K. R. and Meneveau, C., The Fractal Facets of Turbulence, J. Fluid Mech., Vol. 173, (1986), p. 357.

(4) Caswell, W.E. and Yorke, J.A., Fractals in Physics, (1988), p. 123, North-Holland.

(5) Feder, J., Fractals, (1988), p. 6, Plenum Press.

(6) Feder, J. and Aharony, A., Fractals in Physics, (1990), North-Holland.

( 7 ) Tennekes, H. and Lumley, J. L., A First Course in Turbulence, (1972), p. 67, The M. I. T. Press.

(8) Dubuc, B., Quiniou, J. F., Roques-Carmes, C., Tricot, C. and Zucker, S. W., Evaluating the Fractal Dimension of Profiles, Phys. Rev. A., Vol. 39, (1989), p. 1501.

( 9 ) Falconer, K., Fractal Geometry, (1990), p. 36, John Wiley \& Sons.

(10) For example, The Dictionary of Mathematics, 3rd ed., (in Japanese), (1985), $289 \mathrm{H}$, Iwanami.

(11) Shlesinger, M. F., Williams-Watts Dielectric Relaxation: A Fractal Time Stochastic Process, J. Statist. Phys., Vol. 36, No. 5/6, (1984), p. 639.

(12) Sreenivasan, K. R., Prabhu, A. and Narasimha, R., Zero-crossings in Turbulent Signals, J. Fluid Mech. Vol. 137, (1983), p. 251.

(13) Badri Narayanan, M. A., Rajagopalan, S. and Narasimha, R., Experiments on the Fine Structure of Turbulence, J. Fluid Mech., Vol.80, (1977), p. 237.

(14) Mandelbrot, B. B., The Fractal Geometry of Nature, (1983), W. H. Freeman and Company, New York. 